\title{
Features Based on Thin-section Computed Tomography Identify the Type of lung Adenocarcinoma
}

\section{Zhiqiang Li}

Weifang Respiratory Disease Hospital of Shandong

Hongwei Zheng

Weifang Respiratiory Disease Hospital of Shandong

Shanshan Liu

Weifang People's Hospital of Shandong

\section{Xinhua Wang}

Weifang Respiratory Disease Hospital of Shangdong

Lei Xiao

Weifang Respiratory Disease Hospital of Shandong

Yonghong Ma

Shanghai Chest Hospital

Xianwen Yue ( $\nabla$ gowen256@163.com )

Weifang Respiratory Disease Hospital of Shandong

\section{Research article}

Keywords: Thin-section computed tomography, Invasiveness, lung adenocarcinoma, biomarker

Posted Date: March 20th, 2020

DOl: https://doi.org/10.21203/rs.3.rs-18082/v1

License: (c) (i) This work is licensed under a Creative Commons Attribution 4.0 International License. Read Full License 


\section{Abstract}

Background: To investigate whether thin-section computed tomography (TSCT) features may efficiently guide the invasiveness basedclassification of lung adenocarcinoma.

Methods: Totally, 316 lung adenocarcinoma patients (from 2011-2015) were divided into three groups: 56 adenocarcinoma in situ (AIS), 98 minimally invasive adenocarcinoma (MIA), and 162 invasive adenocarcinoma (IAC) according their pathological results. Their TSCT features, including nodule pattern, shape, pleural invasion, solid proportion, border, margin, vascular convergence, air bronchograms, vacuole sign, pleural indentation, diameter, solid diameter, and CT values of ground-glass nodules (GGN) were analyzed. Pearson's chi-square test, Fisher's exact test and One-way ANOVA were adopted tocomparebetweengroups. Receiver operating characteristic (ROC) analysis wereperformedto assess its value for prediction and diagnosis.

Results: Patients with IAC were significantly elder than those in AIS or MIA group,and more MIA patients had a smoking history than AIS and IAC. No recurrence happened in the AIS and MIA groups, while 4.3\% recurrences were confirmed in the IAC group. As for TSCT variables, we found AIS group showed dominantly higher $91.07 \%$ PGGN pattern and $87.50 \%$ round/oval nodules than that in MIA and IAC group. In contrast, MIA group showed more cases with undefined border and vascular convergence than AIS and IAC group. Importantly, IAC group uniquely showed higher frequency of pleural invasion compared with MIA and AIS group. The majority of patients (82.1\%) in IAC group showed $\geq 50 \%$ solid proportion. We found diameter and solid diameter of the lesions were notably larger in the IAC group compared with AIS and MIA groupin quantitative aspect. In addition, for MGGNs, the CT values of ground-glass opacity (GGO) and ground-glass opacity solid portion (GGO-solid) were both higher in the IAC group than AIS and MIA. Finally, we also observed that smooth margin took a dominant proportion in the AIS group while most cases in the IAC group had a lobulate margin. Patients in MIA and IAC group shared higher level of air bronchograms and vacuole signs than AIS group.

Conclusions: The unique features in different groups identified by TSCT had diagnosis value for lung adenocarcinoma.

\section{Background}

Lung adenocarcinoma has been the leading cause of cancer death worldwide [1]. The adenocarcinoma classification protocols had been updated by the International Association for the Study of Lung Cancer (IASLC), the American Thoracic Society (ATS), and the European Respiratory Society (ERS) at 2011( [2, 3]). According to the new international standard, lung adenocarcinoma was divided into AIS, MIA and IAC. AIS and MIA were defined as small (diameter $\leq 3 \mathrm{~cm}$ ) solitary lung adenocarcinoma with lepidic (or at least predominantly lepidic) growth pattern. Tumor cells of MIA grow similarly to AIS, with invasive components $\leq 5 \mathrm{~mm}$, both of which showed lower invasion trait than IAC. 
Early and accurate diagnosis of the type lung carcinoma may largely improve the surgery protocol and medication strategy, and hence benefit the therapy outcomes. CT had been widely used lung adenocarcinoma diagnosis. For example, adenocarcinoma nodules imaged by CT frequently appear as ground-glass nodules (GGN)[4]. The features of adenocarcinoma GGNs have aroused the extensive interests of researchers since the close correlation between those features and tumor types (24851690). To date, low-dose thin-section computed tomography (TSCT) screening was widely used for early lung cancer detection. Previous studiesalso had suggested the value of TSCT features to differentiate among AIS, MIA and IAC. For example, AIS lesions indicate round/oval shape and smooth/notched margin, while MIA lesions exhibit oval/polygonal/irregular shape, shallow notch/lobular/speculated margin[5].

Given CT wasthe necessary step in cancer screening, it was worth to investigate whether TSCT features may guide the lung adenocarcinoma classification.We also analyzedother traits (like recurrence rate, age and smoking history) specifically correlated with AIS/MIA/IAC types. Our work showed that the features, including PGGN pattern, round/oval nodules, border and vascular convergence, pleural invasion, solid proportion. diameter and solid diameter of the lesions, GGO and GGO-solid, smooth margin, air bronchograms and vacuole signs, provideda convincing reference to discriminate cancer types of lung adenocarcinoma.

\section{Methods}

\subsection{Patients}

This prospective study was approved by the Ethic Review Board of the Weifang Respiratory Disease Hospital. Totally, 316 patients (from 2011-2015) were enrolled and the written informed consent forms were obtained from the patients before surgery. The inclusion criterion was all patient withsuspected AIS, MIA or IACs andreceived nodule resection. The exclusion criterion was the patients with synchronous multiple pulmonary nodules (23 excluded cases). The management procedures were strictly accorded with the 2013 guidelines of the Fleischner Society. All the resected nodules were later pathologically determined as either AIS, MIA or IAC. The patients were divided into three groups according to their pathological results. The clinicopathologic factors of the patients were listed in Table 1. The other indexes, such as smoking history and tumor recurrence also were included in the table. 
Table 1

Clinicopathologic features of the enrolled patients

\begin{tabular}{|c|c|c|c|c|c|}
\hline \multicolumn{2}{|l|}{ Variable } & AIS(n) & $\operatorname{MIA}(n)$ & $\operatorname{IAC}(\mathrm{n})$ & p-Value \\
\hline \multicolumn{2}{|l|}{ Age(years) } & $50.1 \pm 11.3$ & $53.4 \pm 13.2$ & $60.4 \pm 11.2$ & $<0.001$ \\
\hline \multirow[t]{2}{*}{ Sex } & Male & 16 & 28 & 59 & \multirow[t]{2}{*}{0.331} \\
\hline & Female & 40 & 70 & 103 & \\
\hline \multirow[t]{3}{*}{ Surgical method } & Wedge & 49 & 55 & 23 & \multirow[t]{3}{*}{-} \\
\hline & Segmentectomy & 2 & 13 & 4 & \\
\hline & Lobar & 5 & 30 & 135 & \\
\hline \multirow[t]{2}{*}{ Smoke } & Yes & 4 & 28 & 17 & \multirow[t]{2}{*}{$<0.001$} \\
\hline & No & 52 & 70 & 145 & \\
\hline
\end{tabular}

\subsection{CT Scan}

CT scan was performed on a Somatom Sensation-64 system (Siemens Healthineers, Erlangen, Germany). The routine CT scan was performed initially with a collimation of $128^{\star} 1.25 \mathrm{~mm}$ and a field of view (FOV) of $500 \mathrm{~mm}$. Once the nodule was found, target TSCT was performed with the following parameters: 64 * $0.625 \mathrm{~mm}$ collimation, 0.64 pitch, $1 \mathrm{~mm}$ section thickness and $1 \mathrm{~mm}$ interval, 0.001 second scan time, $1024 \times 1024$ matrix, $180 \mathrm{~mm}$ FOV, $120 \mathrm{kV}$, and $300 \mathrm{~mA}$.

\subsection{Evaluation Of The TSCT Features And Indexes}

All images were displayed at a mediastinal window (width $350 \mathrm{HU}$, level $35 \mathrm{HU}$ ) using the communication system (Mintech, Beijing, China). The CT data was evaluated by two independent chest radiologists in a blind method, and all personal information had been erased before reading. In discrepancy cases, a third radiologist was consulted. The following CT characteristics were obtained [6]: (1) nodule patterns: pure ground-glass nodule (PGGN) or mixed ground-glass nodule (MGGN); (2) nodule shapes (round/oval shape or irregular shape); (3) pleural invasion (or not); (4) vascular invasion (or not); (5) lymphatic invasion (or not); (6) necrosis (or not); (7) diameter of the nodule (largest diameter on the axial section); (8) diameter of the solid component (largest diameter of solid component on the axial section; (9) proportion of the solid component $(<50 \%$ or $>50 \%)$; $(10)$ the border of the nodule was defined or undefined; (11) the margin of the nodule (smooth, lobulate, or spiculate); (12) vascular convergence sign (or not); (13) air-bronchograms (direct involvement of bronchiole, in the GGO component or in the solid 
component, no); (14) vacuole sign (or not, point-like/translucent/low-density shadows); (15) pleural indentation (or not). Besides, CT radiodensity values were recorded (for PGGN, the value of GGO component; for MGGN, the value of solid component).

\subsection{Histopathological Identification}

Before surgery, radiologists, thoracic surgeons, and pathologists discussed and decided the best strategies. Lesions were surgically removed, by which the specimens were obtained. For pathological identification, specimens were fixed in formalin (10\%, for $24 \mathrm{~h}$ ) and then embedded by paraffin. After hematoxylin-eosin (H\&E) staining, sections were observed under microscope. Histological types were classified according to the IASLC/ATS/ERS classification of lung adenocarcinoma. All histological analyses were performed by two senior pathologists, and a third pathologist was consulted in any case of disagreement.

\subsection{Statistical Analysis}

Qualitative variables (such as nodule pattern, nodule shape, nodule border) were assessed by Pearson's chi-square test and Fisher's exact test. Quantitative variables were expressed as Mean \pm Standard Deviation and compared by One-way ANOVA. The significant variables were further analyzed by multivariate logistic regression analysis and receiver operating characteristic (ROC) analysis to assess the values for prediction and diagnosis. SPSS (IBM, Armonk, NY, USA) was applied for analysis. A value of $p<0.05$ was considered statistically significant.

\section{Results}

\subsection{Clinicopathologic Features of Patients}

As shown in Table 1, a total of 316 patients were enrolled, among which 56 (17.7\%) were in the AIS group, 98 (31.0\%) in the MIA and $162(51.3 \%)$ in the IAC group. Females were dominant but no statistical difference was found in the sex composition $(p=0.331)$. Patients in IAC group were significantly elder than those in AIS or MIA groups $(p<0.001)$ while the AIS and MIA groups had a similar age range, indicating the positive correlation between age and invasiveness of lung adenocarcinoma. The majority (83.3\%) of the IAC patients received the lobar resection, while the overwhelming percentage (87.5\%)of AIS patients underwent wedge resection. However, the resection type in MIA patients showed no obvious predilection. Noteworthily, $29 \%$ of the MIA group had a smoke history, compared with the $10 \%$ in the IAC group and $7 \%$ in the AIS group. This result suggested that smoke is positively correlated with the onset of lung adenocarcinoma but show no obvious effect on invasive evolution during tumor progression.

\subsection{HRCT Morphological Features Of Different Groups}


As shown in Table 2, the HRCT morphological features exhibited significant differences among the three groups. In the AIS group, the frequency of PGGN pattern was dominantly high (91.07\%). However, the PGGN frequency dramatically dropped to $60.20 \%$ and $4.94 \%$ in MIA and IAC group respectively $(p<0.001$, with a highest $\chi 2$ value among all characteristics). 
Table 2

HRCT Morphological features of different groups.

\begin{tabular}{|c|c|c|c|c|c|c|}
\hline Features & & AIS(n) & MIA(n) & $\operatorname{IAC}(\mathrm{n})$ & $x^{2}$ & p-Value \\
\hline \multirow[t]{2}{*}{ Pattern } & PGGN & 51 & 59 & 8 & \multirow[t]{2}{*}{163.9} & \multirow[t]{2}{*}{$<0.001$} \\
\hline & MGGN & 5 & 39 & 154 & & \\
\hline \multirow[t]{2}{*}{ Shape } & Round/oval & 49 & 84 & 100 & \multirow[t]{2}{*}{24.8} & \multirow[t]{2}{*}{$<0.001$} \\
\hline & Irregular & 7 & 14 & 62 & & \\
\hline \multirow[t]{2}{*}{ Pleural invasion } & + & 0 & 0 & 31 & \multirow[t]{2}{*}{32.7} & \multirow[t]{2}{*}{$<0.001$} \\
\hline & - & 56 & 98 & 131 & & \\
\hline \multirow[t]{2}{*}{ Vascular invasion } & + & 0 & 0 & 3 & \multirow[t]{2}{*}{2.88} & \multirow[t]{2}{*}{0.237} \\
\hline & - & 56 & 98 & 159 & & \\
\hline \multirow[t]{2}{*}{ Lymphatic invasion } & + & 56 & 98 & 156 & \multirow[t]{2}{*}{5.81} & \multirow[t]{2}{*}{0.550} \\
\hline & - & 0 & 0 & 6 & & \\
\hline \multirow[t]{2}{*}{ Necrosis } & + & 56 & 97 & 160 & \multirow[t]{2}{*}{0.68} & \multirow[t]{2}{*}{0.711} \\
\hline & - & 0 & 1 & 2 & & \\
\hline \multirow[t]{2}{*}{ Solid proportion } & $<50 \%$ & 56 & 89 & 30 & \multirow[t]{2}{*}{184} & \multirow[t]{2}{*}{$<0.001$} \\
\hline & $\geq 50 \%$ & 0 & 9 & 132 & & \\
\hline \multirow[t]{2}{*}{ Border } & Defined & 53 & 85 & 155 & \multirow[t]{2}{*}{7.61} & \multirow[t]{2}{*}{0.022} \\
\hline & Undefined & 3 & 13 & 7 & & \\
\hline \multirow[t]{3}{*}{ Margin } & Smooth & 39 & 64 & 22 & \multirow[t]{3}{*}{99.64} & \multirow[t]{3}{*}{$<0.001$} \\
\hline & Lobulate & 12 & 34 & 115 & & \\
\hline & Spiculate & 5 & 0 & 25 & & \\
\hline \multirow[t]{2}{*}{ Vascular convergence } & + & 15 & 70 & 68 & \multirow[t]{2}{*}{33.96} & \multirow[t]{2}{*}{$<0.001$} \\
\hline & - & 41 & 28 & 94 & & \\
\hline \multirow[t]{3}{*}{ Air bronchograms } & Without & 54 & 16 & 38 & \multirow[t]{3}{*}{219.42} & \multirow[t]{3}{*}{$<0.001$} \\
\hline & GGO & 2 & 67 & 22 & & \\
\hline & Solid & 0 & 15 & 101 & & \\
\hline $\begin{array}{l}\text { AIS: adenocarcinoma i } \\
\text { adenocarcinoma. }\end{array}$ & „; MIA: minima & invasive & lenocarci & ma; IAC: & asive & \\
\hline
\end{tabular}




\begin{tabular}{|lcccccc|}
\hline Features & AIS(n) & MIA(n) & IAC(n) & X2 & p-Value \\
\hline Vacuole sign & + & 6 & 24 & 116 & 89.02 & $<0.001$ \\
\cline { 2 - 6 } & - & 50 & 74 & 46 & & \\
Pleural indentation & + & 1 & 21 & 126 & 133.34 & $<0.001$ \\
\hline $\begin{array}{l}\text { AlS: adenocarcinoma in situ; MIA: minimally invasive adenocarcinoma; IAC: invasive } \\
\text { adenocarcinoma. }\end{array}$ & & & & & \\
\hline PGGN: pure ground-glass nodule; MGGN: mixed ground-glass nodule; GGO: Ground-glass opacity. & \\
\hline
\end{tabular}

The AIS group had $87.50 \%$ round/oval nodules, which decreased to $85.7 \%$ and $61.7 \%$ in MIA and IAC group, suggesting that the invasive lung adenocarcinoma tend to be shaped irregularly $(p<0.001)$. Besides, IAC lesions showed higher frequency of pleural invasion compared other two groups $(p<0.001)$, while no statistical differences in the vascular invasion and lymphatic invasion and tumor necrosis status were found among three groups. The majority of patients (82.1\%) in IAC group showed $\geq 50 \%$ solid proportion. In sharp contrast, only $9.2 \%$ in MIA group and none in AIS group exhibited $\geq 50 \%$ solid proportion. Further, MIA group showed more cases with undefined border than AIS and IAC $(p<0.05)$. We also observed that smooth margin took a dominant proportion In the AIS group while most cases in the IAC group had a lobulate margin $(p<0.001)$. It is notable that the vascular convergence was rare in the AIS $(26.8 \%)$ group, and it exhibited gradient increase from IAC (41.9\%) to MIA(71.4\%) group.

In addition, patients in MIA and IAC group showed higher level of air bronchograms and vacuole signs than AIS group ( $p<0.001$, respectively). Finally, another important characteristic with a high $\chi 2$ was whether there exists the pleural indentation, which showed a high probability in IAC (77.8\%) cases but hardly in AIS $(1.8 \%)(p<0.001)$.

\subsection{HRCT Quantitative Features Of Different Groups}

As shown in Table 3, we found that the diameter and solid diameter of the lesions were notably larger in the IAC group compared to AIS and MIA $(p<0.001)$, and no statistical difference between AIS and MIA. For MGGNs, the CT values of ground-glass opacity (GGO) and ground-glass opacity solid portion (GGOsolid) were both higher in the IAC group than AIS and MIA ( $p<0.001)$. In contrast, we observed no statistical different of CT radiodensity values of PGGNs among the three groups. 
Table 3

Qualitative features of different groups in HRCT

\begin{tabular}{|llllll|}
\hline Features & AIS & MIA & IAC & F & p-Value \\
\hline Diameter $(\mathrm{mm})$ & $\begin{array}{l}9.7 \pm 5.8 \\
(\mathrm{n}=56)\end{array}$ & $\begin{array}{l}11.0 \pm 5.0 \\
(\mathrm{n}=98)\end{array}$ & $\begin{array}{l}21.5 \pm 5.6 \\
(\mathrm{n}=162)\end{array}$ & 159.6 & $<0.001$ \\
\hline Solid Diameter $(\mathrm{mm})$ & $\begin{array}{l}6.8 \pm 1.1 \\
(\mathrm{n}=5)\end{array}$ & $\begin{array}{l}5.7 \pm 2.1 \\
(\mathrm{n}=34)\end{array}$ & $\begin{array}{l}14.4 \pm 5.7 \\
(\mathrm{n}=154)\end{array}$ & 47.2 & $<0.001$ \\
\hline CT value-PGGN & $-624.2 \pm 91.2$ & $-567.2 \pm 123.3$ & $-589.9 \pm 136.4$ & 1.998 & 0.140 \\
\hline CT value-MGGN (GGO) & $-536.2 \pm 192.9$ & $-582.3 \pm 100.7$ & $-458.1 \pm 142.5$ & 13.2 & $<0.001$ \\
\hline CT value-MGGN (solid) & $-592.2 \pm 106.7$ & $-320.8 \pm 175.5$ & $-47.08 \pm 123.2$ & 96.43 & $<0.001$ \\
\hline $\begin{array}{l}\text { AIS: adenocarcinoma in situ; MIA: minimally invasive adenocarcinoma; IAC: invasive } \\
\text { adenocarcinoma. }\end{array}$ & & & & \\
\hline \multicolumn{4}{l}{ PGGN: pure ground-glass nodule; MGGN: mixed ground-glass nodule; GGO: Ground-glass opacity. } \\
\hline
\end{tabular}

\subsection{ROC Curve Analysis Of IAC Group}

The diagnostic performances of above variables that exhibited unique features in IAC group was analyzed by receiver operating characteristic (ROC) analyses (Fig. 2). The CT value of MGGN (solid component) exhibited the largest area (0.917). Important cutoffs were as follow: CT value-MGGN (solid) $=-107 \mathrm{HU}$, sensitivity $=77 \%$, specificity $=93 \%$; Diameter $=14.7 \mathrm{~mm}$, sensitivity $=90 \%$, specificity $=81 \%$ (Table 4).

Table 4

The parameters for ROC analysis.

\begin{tabular}{|lllll|}
\hline Features & value & sensitivity & specificity & AUC \\
\hline MGGN(solid component) & $-107 \mathrm{HU}$ & $77 \%$ & $93 \%$ & 0.917 \\
\hline Diameter & $14.7 \mathrm{~mm}$ & $90.0 \%$, & $81.0 \%$ & 0.849 \\
\hline C-Marker & 0.287 & $93.5 \%$ & $97.7 \%$ & 0.982 \\
\hline Margin & & $83.9 \%$ & $81.0 \%$ & 0.779 \\
\hline Air bronchograms & $74.6 \%$ & $79.7 \%$ & 0.744 \\
\hline $\begin{array}{l}\text { MGGN: mixed ground-glass nodule; C-Marker: combined marker, a ROS model to comprehensively } \\
\text { evaluate the correlation between parameters; AUC: Area under the curve. }\end{array}$ & \\
\hline
\end{tabular}

Further, we developeda linear regression model and acquired a novel factor (named Combined Marker, or C-Maker) with 13 variables: $\mathrm{C}$-marker $=0.068^{*}$ shape $-0.063^{*}$ pleural invasion $+0.326 *$ solid proportion - 
0.056 border $-0.214^{\star}$ margin $+0.077^{*}$ vascular convergence $+0.181^{*}$ air bronchograms $-0.124 *$ vacuole sign + 0.027 * pleural indentation + 0.001 * CT value of MGGN (solid) + 4.352e-005* CT value of MGGN $(G G O)+0.01 *$ diameter -0.004 * solid diameter. Here, CT value of MGGN (GGO), CT value of MGGN (solid), diameter and solid diameter were numerical variables and other variables were categorical data (encoded by 0 or 1 and 1 to 3, as shown in Table 2). Again, we draw a ROC curve using C-marker, which exhibited an AUS as high as 0.982 (Fig. 2). The cutoff value for IAC was 0.287 , with a sensitivity of 0.935 and specificity of 0.977 , which could be strongly recommended in determination of lung carcinoma classification(Table 4).

\subsection{The Recurrence Rate Of Patients}

We analyzed the recurrence rate after two years' follow up and found significant difference among the three groups $(\chi 2=6.805, p=0.033)$. No recurrence happened in the AIS and MIA groups, while 7 cases (4.3\%) suffered recurrence in the IAC group. This is consistent with previous knowledge that IAC cancer type had a higher malignancy and poorer prognosis.

\section{Discussion}

Accurate diagnosis of lung adenocarcinoma type could efficiently improve the appropriate surgery and medication strategy, and hence benefit the therapy outcomes. Here, we tend to investigate novel features to make accurate classification among AIS, MIA, IAC. We found that age is a contributor to IAC development and smoking is positively correlated with the occurrence of MIA. Importantly, the unique features in different groups identify by TSCT had diagnosis value for lung adenocarcinoma.

Our results are consistent with previous studies. Shi et al revealed that there existed huge differences in the CT measurements of solitary GGNs between AIS-MIA and IAC [7]. Different from our study, they combined the AIS and MIA into one group. Their work also pointed out that nodule types, cystic appearances, diameters and CT values were different between groups, despite no differences were found in shapes, margins, bronchus sign, pleural indentation. These inconsistencies maybe due to different grouping method and different sample size (their sample size was much smaller than ours). In contrast, Another research showed that the unique feature of shape and margin could be used to discriminate different types of carcinoma types([8] [9]). Besides, patients in IAC group showed higher frequency of air bronchogram and pleural indentation, and CT values (HU) than AIS-MIA group [2]. Consistently, homma et al recently found that malignant signs could include lobulation, spiculation, vascular convergence and pleural indentation in invasive mucinous adenocarcinoma [10]. Although their sample size was small, their conclusion highly supported our results.

The correlation between age, smoking and lung adenocarcinoma types is controversial. We here believe that age is a contributor to IAC development, and smoking is positively correlated with MIA formation. Consistently, one group also found that ages are comparable between AIS and MIA patients but showed correlation with the occurrence of IAC [11]. In contrast, it was also reported that age and smoking showed 
no obvious correlation with lung adenocarcinoma types[12]. Our results implied that more treatment should be adopted to inhibit tumor invasion for elder patients. As for the features of smoking, we paradoxically found that smoking ratio increased in MIA compared with AIS but dropped in IAC group. A pooled estimation only showed weak effect of secondhand tobacco smoke exposure on AIS/MIA incidence [13], and another study also observe no correlation between smoking history and invasive carcinoma [14]. However, it was also reported that tobacco exposure may accelerate malignant progression [15], and smoking is not a major cause of AIS but play a role in the progression from AIS to the invasive adenocarcinoma still with AIS features [16].

We found that patients in ICA showed unique features of higher solid or GGO components and diameter of GGN. The solid components can be firosis, invasive component, collapsed alveolar or other parenchyma, which was related to fibrotic focus, alveoli collapse and tumor cell proliferation [17]. It was believed that GGO-dominant tumors could be identified as minimally invasive and with recent 5-year survival rates exceeding $90 \%$ [18]. In contrast, the majority of AIS and MIA cases had a solid proportion less than $50 \%$. As Fig. 2 showed, the solid components in MGGN is the most powerful variable to identify IAC patients. The CT value of solid or GGO component is also impacted by air cavity densities, which include air bronchograms and vacuole sign. Air bronchograms in MIA commonly present distortion and extension in the GGO component. In this study, we found that air bronchograms in GGO component was dramatically higher in the MIA group than the other two groups, and thus showed an obviously predictive value to MIA. In addition, vacuole sign is another factor belonging to air cavity densities, as well as a useful indicator for IAC in our study. There is no existing evidence about the diagnostic role the vacuole sign in adenocarcinoma recognition. Together, besides CT values of solid or GGO components and diameters, the vacuole sign and air bronchograms might be supplemental features in predicting the lung adenocarcinoma types.

More importantly, we analyzed the diagnostic of GGO components, diameters, the vacuole sign and air bronchograms by receiver operating characteristic (ROC) analyses to convincingly testified the correlation between those parameters and IAC types. Further, we also adopted a linear regression model, C-Maker, to comprehensively evaluated the role played by 13 variables. Noteworthily, we draw a ROC curve using Cmarker, which exhibited an AUS as high as 0.982 . Its cutoff value for IAC was 0.287 , with a sensitivity of 0.935 and specificity of 0.977 , indicating that those variables was applicable in the determination of lung carcinoma type.

This study has some limitations. First, the sample size of three groups was still not very large. Or else we can perform a principal component analysis and then choose the most conclusive parameters to carry out machine learning, which may predict the probability of each subtype for any specific patient. Second, we demonstrated smoking is positively correlated with MIA formation, but the mechanism underlying the higher smoking rate in MIA group while IAC paradoxically had a lower rate is still confusing. Third, smoking is an important variable related to adenocarcinoma clarifications (especially for MIA), but the smoking habits of the smokers were not detailed or presented in pack-years. If more details were recorded, more convincing correlations might be probed. 


\section{Conclusion}

In conclusion, the unique TSCT morphological features, including PGGN pattern, round/oval nodules, border and vascular convergence, pleural invasion, solid proportion. diameter and solid diameter of the lesions, GGO and GGO-solid, smooth margin, air bronchograms and vacuole signs, in different group had predictive meanings in discriminating three adenocarcinoma types before surgery and thus guided thestrategy of nodule resection and further treatments.

\section{Abbreviations}

AIS

Adenocarcinoma in situ;

ATS

American Thoracic Society;

FOV

Field of view;

GGN

Ground-glass nodules;

H\&E

Hematoxylin-eosin;

IASLC

International Association for the Study of Lung Cancer;

IAC

Invasive adenocarcinoma;

GGO

Ground-glass opacity;

MGGN

Mixed ground-glass nodule;

MIA

Minimally invasive adenocarcinoma;

PGGN

Pure ground-glass nodule;

ROC

Receiver operating characteristic;

TSCT

Thin-section computed tomography

\section{Declarations}

Ethics approval and consent to participate: Not applicable 
Consent for publication: Not applicable

Availability of data and materials: Not applicable

Competing interests: The authors declare that they have no competing interests

Funding: Not applicable

Acknowledgements: Not applicable

\section{References}

1. Pastorino U. Current status of lung cancer screening. Thorac Surg Clin 2013;23:129-40.

2. Lynch DA, Austin JH, Hogg JC, Grenier PA, Kauczor HU, Bankier AA, Barr RG, Colby TV, Galvin JR, Gevenois PA, Coxson HO, Hoffman EA, Newell JD, Jr., Pistolesi M, Silverman EK, Crapo JD. CTDefinable Subtypes of Chronic Obstructive Pulmonary Disease: A Statement of the Fleischner Society. Radiology 2015;277:192-205.

3. Naidich DP, Bankier AA, MacMahon H, Schaefer-Prokop CM, Pistolesi M, Goo JM, Macchiarini P, Crapo JD, Herold CJ, Austin JH, Travis WD. Recommendations for the management of subsolid pulmonary nodules detected at CT: a statement from the Fleischner Society. Radiology 2013;266:304-17.

4. Gao F, Ge XJ, Li M, Chen Y, Lyu F, Hua Y, Ren Q, Qi L. [CT diagnosis of different pathological types of ground-glass nodules]. Zhonghua Zhong Liu Za Zhi 2014;36:188-92.

5. Lee HJ, Goo JM, Lee CH, Park CM, Kim KG, Park EA, Lee HY. Predictive CT findings of malignancy in ground-glass nodules on thin-section chest CT: the effects on radiologist performance. Eur Radiol 2009;19:552-60.

6. Bankier AA, MacMahon H, Goo JM, Rubin GD, Schaefer-Prokop CM, Naidich DP. Recommendations for Measuring Pulmonary Nodules at CT: A Statement from the Fleischner Society. Radiology 2017;285:584-600.

7. Zhou J, Li Y, Zhang Y, Liu G, Tan H, Hu Y, Xiao J, Shi H. Solitary ground-glass opacity nodules of stage IA pulmonary adenocarcinoma: combination of 18F-FDG PET/CT and high-resolution computed tomography features to predict invasive adenocarcinoma. Oncotarget 2017;8:2331223321.

8. Zhang Y, Qiang JW, Ye JD, Ye XD, Zhang J. High resolution CT in differentiating minimally invasive component in early lung adenocarcinoma. Lung Cancer 2014;84:236-41.

9. Zhang Y, Shen Y, Qiang JW, Ye JD, Zhang J, Zhao RY. HRCT features distinguishing pre-invasive from invasive pulmonary adenocarcinomas appearing as ground-glass nodules. Eur Radiol 2016;26:29218.

10. Miyamoto A, Kurosaki A, Fujii T, Kishi K, Homma S. HRCT features of surgically resected invasive mucinous adenocarcinoma associated with interstitial pneumonia. Respirology 2017;22:735-743. 
11. Liu J, Li W, Huang Y, Mu D, Yu H, Li S. [Differential diagnosis of the MDCT features between lung adenocarcinoma preinvasive lesions and minimally invasive adenocarcinoma appearing as groundglass nodules]. Zhonghua Zhong Liu Za Zhi 2015;37:611-6.

12. Si MJ, Tao XF, Du GY, Cai LL, Han HX, Liang XZ, Zhao JM. Thin-section computed tomographyhistopathologic comparisons of pulmonary focal interstitial fibrosis, atypical adenomatous hyperplasia, adenocarcinoma in situ, and minimally invasive adenocarcinoma with pure groundglass opacity. Eur J Radiol 2016;85:1708-1715.

13. Kim CH, Lee YC, Hung RJ, Boffetta P, Xie D, Wampfler JA, Cote ML, Chang SC, Ugolini D, Neri M, Le Marchand L, Schwartz AG, Morgenstern H, Christiani DC, Yang P, Zhang ZF. Secondhand Tobacco Smoke Exposure and Lung Adenocarcinoma In Situ/Minimally Invasive Adenocarcinoma (AIS/MIA). Cancer Epidemiol Biomarkers Prev 2015;24:1902-6.

14. Rezaee N, Khalifian S, Cameron JL, Pawlik TM, Hruban RH, Fishman EK, Makary MA, Lennon AM, Wolfgang CL, Weiss MJ. Smoking is not associated with severe dysplasia or invasive carcinoma in resected intraductal papillary mucinous neoplasms. J Gastrointest Surg 2015;19:656-65.

15. Carr RA, Roch AM, Shaffer K, Aboudi S, Schmidt CM, 2nd, DeWitt J, Ceppa EP, House MG, Zyromski NJ, Nakeeb A, Schmidt CM. Smoking and IPMN malignant progression. Am J Surg 2017;213:494497.

16. Sato S, Motoi N, Hiramatsu M, Miyauchi E, Ono H, Saito Y, Nagano H, Ninomiya H, Inamura K, Uehara $\mathrm{H}$, Mun M, Sakao Y, Okumura S, Tsuchida M, Ishikawa Y. Pulmonary adenocarcinoma in situ: analyses of a large series with reference to smoking, driver mutations, and receptor tyrosine kinase pathway activation. Am J Surg Pathol 2015;39:912-21.

17. Gandara DR, Aberle D, Lau D, Jett J, Akhurst T, Heelan R, Mulshine J, Berg C, Patz EF, Jr. Radiographic imaging of bronchioloalveolar carcinoma: screening, patterns of presentation and response assessment. J Thorac Oncol 2006;1:S20-6.

18. Asamura H, Hishida T, Suzuki K, Koike T, Nakamura K, Kusumoto M, Nagai K, Tada H, Mitsudomi T, Tsuboi M, Shibata T, Fukuda H, Japan Clinical Oncology Group Lung Cancer Surgical Study G. Radiographically determined noninvasive adenocarcinoma of the lung: survival outcomes of Japan Clinical Oncology Group 0201. J Thorac Cardiovasc Surg 2013;146:24-30.

\section{Figures}



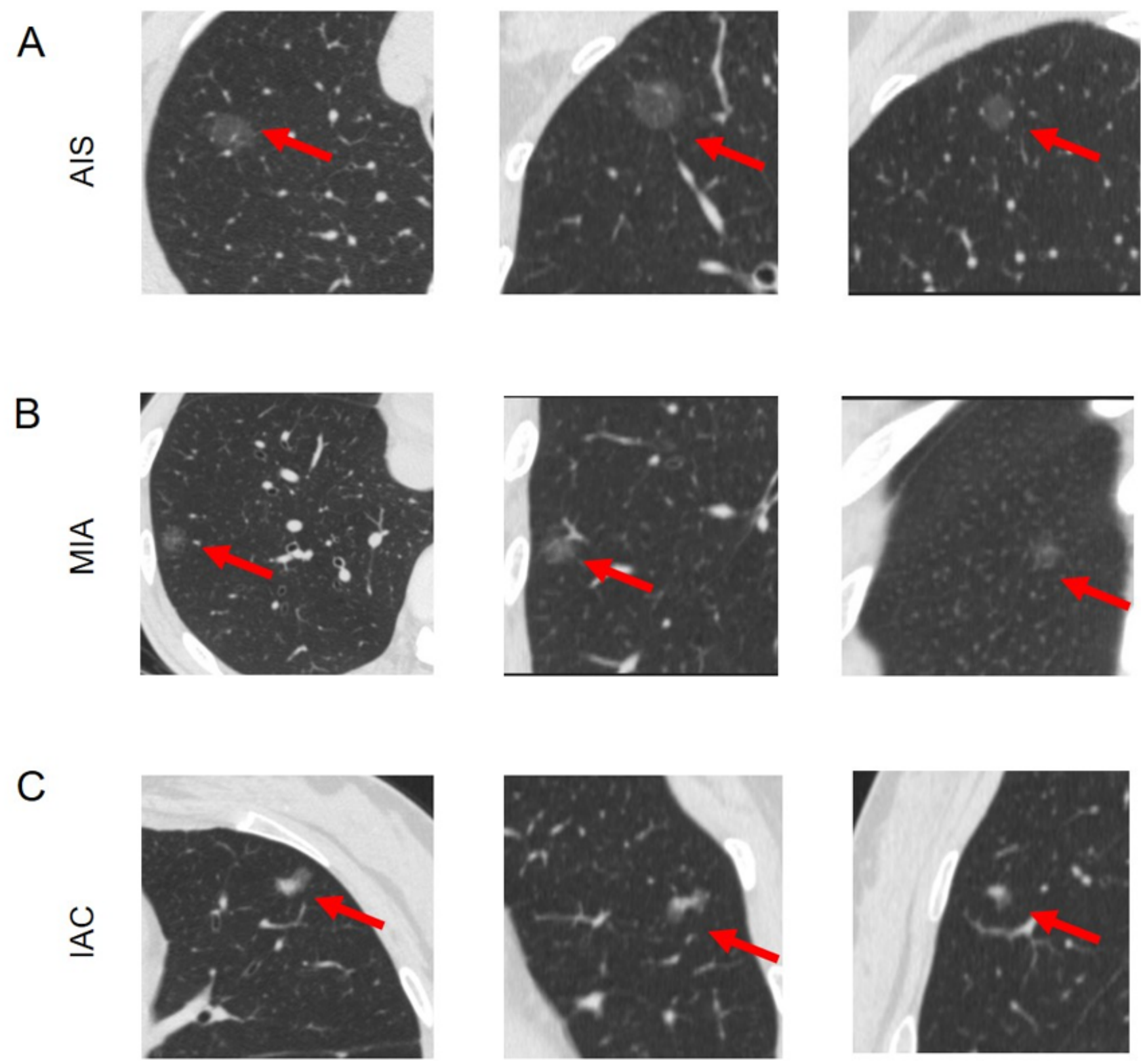

\section{Figure 1}

The representative HRCT images of theAIS, MIA or IAC cases. The left is the axial image; the centered is the coronal image; the right is the sagittal image. The MIA lesion is labeled by a red arrow. (A) the typical AIS case. This patient was a 48-year-old female, with purely ground glass nodules in the upper right lobe of the right lung. The maximum cross-sectional diameter of the lesion was approximately $1 \mathrm{~mm} * 7 \mathrm{~mm}$ (CT value: $-731 \mathrm{HU}$ ). (B) The typical MIA case. This patient was a 63-year-old female, with a purely ground glass nodule in the basal segment of the right lung lobe. Intralesional microangiographic sign and vacuole sign could be observed. The maximum cross-sectional diameter of the lesion was approximately $12 \mathrm{~mm}$ * $10 \mathrm{~mm}$ (CT value: $-574 \mathrm{HU}$ ). (C) The typical IAC case. This patient was a 36-year-old female, with mixed glass nodules in the upper lobe of the left lung. The solid proportion was higher than $50 \%$. A 
lobulate margin could be observed. The maximum cross-sectional diameter of the lesion was approximately $13 \mathrm{~mm} * 9 \mathrm{~mm}$ (CT value: $-181 \mathrm{HU}$ in the solid ground and $-634 \mathrm{HU}$ in the glass ground).

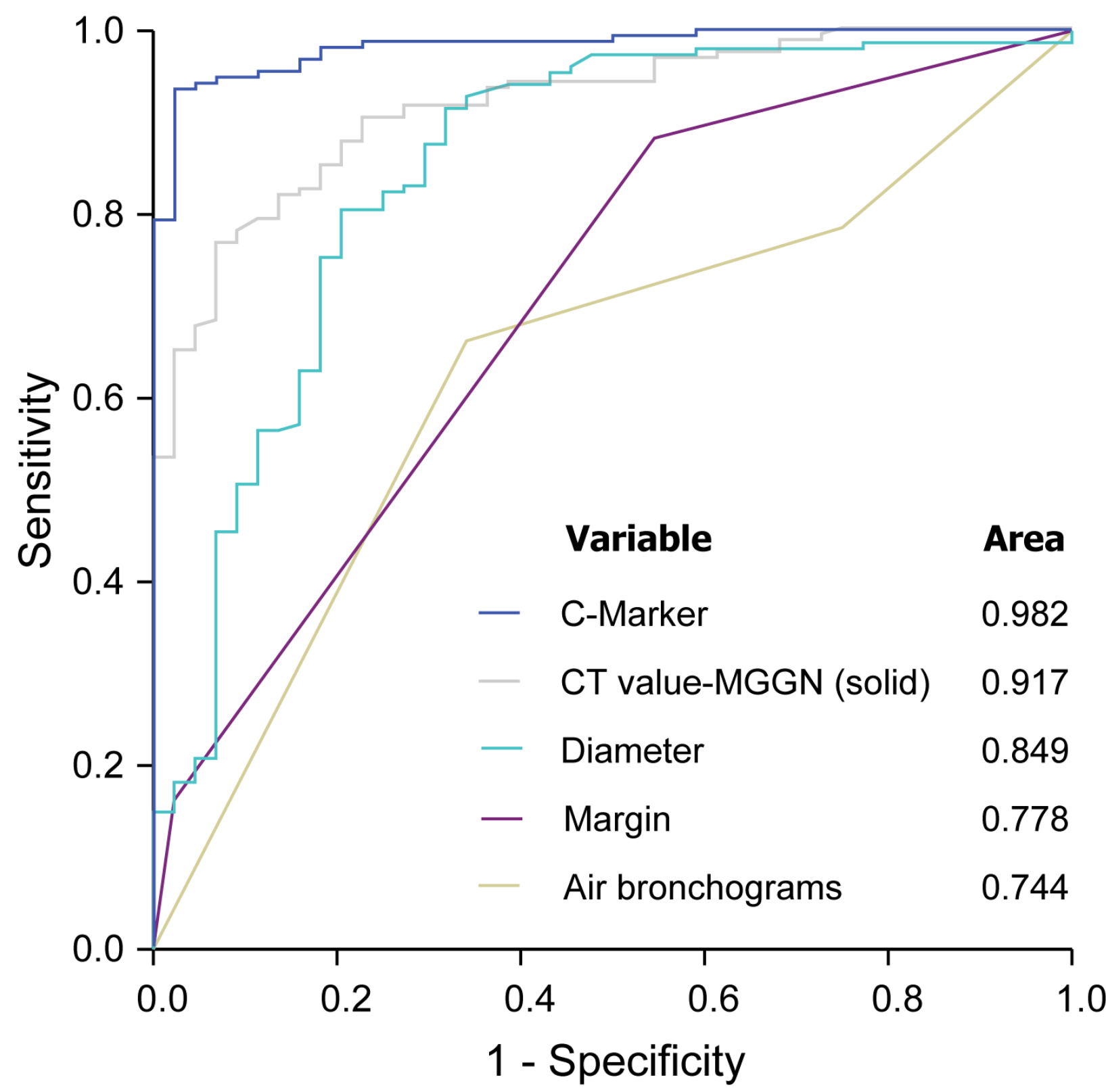

Figure 2

ROC curves of important variables for IAC prediction. The figure included not only traditional features but also a novel marker (C-Marker) calculated with 13 variables whichexhibited an AUC as high as 0.982 . Its cutoff value for IAC was 0.287 , with a sensitivity of 0.935 and specificity of 0.977 . 